\title{
Presence of invasive cribriform or intraductal growth at biopsy outperforms percentage grade 4 in predicting outcome of Gleason score $3+4=7$ prostate cancer
}

Charlotte F Kweldam ${ }^{1}$, Intan P Kümmerlin ${ }^{1}$, Daan Nieboer ${ }^{2}$, Ewout W Steyerberg ${ }^{2}$, Chris $\mathrm{H}$ Bangma ${ }^{3}$, Luca Incrocci ${ }^{4}$, Theodorus $\mathrm{H}$ van der Kwast ${ }^{5}$, Monique J Roobol ${ }^{3}$ and Geert J van Leenders ${ }^{1}$

${ }^{1}$ Department of Pathology, Erasmus Medical Centre, Josephine Nefkens Institute, Rotterdam, The Netherlands; ${ }^{2}$ Department of Public Health, Erasmus Medical Centre, Rotterdam, The Netherlands; ${ }^{3}$ Department of Urology, Erasmus Medical Centre, Rotterdam, The Netherlands; ${ }^{4}$ Department of Radiotherapy, Erasmus Medical Centre, Rotterdam, The Netherlands and ${ }^{5}$ Laboratory Medicine Program, University Health Network, Toronto, ON, Canada

\begin{abstract}
Relative increase of grade 4 and presence of invasive cribriform and/or intraductal carcinoma have individually been associated with adverse outcome of Gleason score 7 (GS 7) prostate cancer. The objective of this study was to investigate the relation of Gleason grade 4 tumor percentage (\%GG4) and invasive cribriform and/or intraductal carcinoma in GS $3+4=7$ prostate cancer biopsies. We reviewed 1031 prostate cancer biopsies from the European Randomized Study of Screening for Prostate Cancer. In total 370 men had G3+4 = 7. The relation of invasive cribriform and/or intraductal carcinoma and \%GG4 with biochemical recurrence-free survival (BCRFS) after radical prostatectomy $(n=146)$ and radiation therapy $(n=195)$ was analyzed using Cox regression. Invasive cribriform and/or intraductal carcinoma occurred in 7/121 (6\%) patients with 1-10\% GG4, 29/131 (22\%) with 10$25 \%$, and $52 / 118(44 \%)$ with $25-50 \%$ GG4 $(P<0.001)$. In crude analysis, both invasive cribriform and/or intraductal carcinoma (HR 2.72; 95\% Cl: 1.33-5.95; $P=0.006$ ) and 10-50\% GG4 (HR 2.43; 95\% Cl: 1.10-5.37; $P=0.03$ ) were associated with BCRFS after prostatectomy. In adjusted analysis, invasive cribriform and/or intraductal carcinoma was an independent predictor for BCRFS (HR 2.40; 95\% Cl: 1.03-5.60; $P=0.04$ ) after prostatectomy, whereas percentage \%GG4 (HR 1.00; 95\% Cl: 0.97-1.03; $P=0.80$ ) was not. While invasive cribriform and/or intraductal carcinoma (HR 2.58; 95\% Cl: 1.59-4.21; $P<0.001$ ) performed better than $10-50 \%$ GG4 (HR 1.24; $95 \%$ $\mathrm{Cl}$ : 0.67-2.29; $P=0.49$ ) for prediction of BCRFS after radiation therapy, both parameters were insignificant in analysis adjusted for prostate-specific antigen $(P=0.001)$, positive biopsies $(P<0.001)$ and tumor volume $(P=0.05)$. In conclusion, increased \%GG4 is associated with invasive cribriform and/or intraductal carcinoma in GS $3+4=7$ prostate cancer biopsies. Invasive cribriform and/or intraductal carcinoma is an independent parameter for BCR after prostatectomy, whereas \%GG4 is not. The presence of invasive cribriform and/or intraductal carcinoma has to be included in pathology reports and should act as exclusion criterion for active surveillance.
\end{abstract}

Modern Pathology (2017) 30, 1126-1132; doi:10.1038/modpathol.2017.29; published online 19 May 2017

The Gleason score (GS) is an important pathologic parameter for risk stratification and therapeutic decision making in prostate cancer patients. While many patients with GS 6 are eligible for surveillance,

Correspondence: Dr GJ van Leenders, MD, PhD, Department of Pathology, Erasmus Medical Centre, Josephine Nefkens Institute, Room Be-222, PO Box 2040, Rotterdam 3000 CA, The Netherlands. E-mail: g.vanleenders@erasmusmc.nl

Received 9 August 2016; revised 17 January 2017; accepted 28 February 2017; published online 19 May 2017 active treatment is generally preferred in men with GS $3+4=7$ cancer. GS $3+4=7$ prostate cancer shows considerable heterogeneity in pathologic features, molecular background, and clinical outcome. For optimal individual therapeutic decision making, therefore, risk stratification of men with GS $3+4=7$ prostate cancer is crucial.

As GS at biopsies is determined by adding the predominant and highest Gleason grade, GS $3+4=7$ encompasses cancer with variable quantities of Gleason grade 4 patterns ranging from $<5 \%$ to up 
to $50 \% .^{1,2}$ The risk of biochemical recurrence (BCR) after radical prostatectomy is incremental with the percentage of Gleason pattern 4 at the surgical specimen. ${ }^{3-5}$ On the other hand, it has been shown that cribriform pattern Gleason grade 4 is associated with BCR and disease-specific death. ${ }^{5-9}$ Finally, the presence of intraductal carcinoma of the prostate (IDC-P), representing a malignant epithelial proliferation within pre-existent dilated glandular structures, is a marker for aggressive disease behavior. ${ }^{7,9-11}$ Invasive cribriform Gleason grade 4 prostate cancer and intraductal carcinoma show overlapping morphologic features, and might be difficult to differentiate without basal cell immunohistochemistry. For practical purposes, therefore, we have labeled invasive cribriform and intraductal growth pattern as one group. ${ }^{7}$

While Gleason grade 4 tumor percentage (\%GG4) and presence of invasive cribriform and/or intraductal carcinoma are both of clinical importance for risk stratification of GS $3+4=7$ patients, it is unclear how both pathologic parameters are related and whether they both provide independent clinical information. The aim of the current study was to analyze the relation between both parameters on diagnostic biopsies, and to determine their relevance in predicting clinical outcome in GS $3+4=7$ prostate cancer patients.

\section{Materials and methods}

\section{Patient Selection}

We included all 1078 men from the first screening round of the Dutch part of the European Randomized Study of Screening for Prostate Cancer (ERSPC), who had been diagnosed with prostate cancer between November 1993 and March 2000 in Erasmus Medical Centre, Rotterdam, The Netherlands. The trial protocol has been published previously. ${ }^{12,13}$ After pathologic review of all available slides $(n=1054)$ and exclusion of men with metastatic disease at the
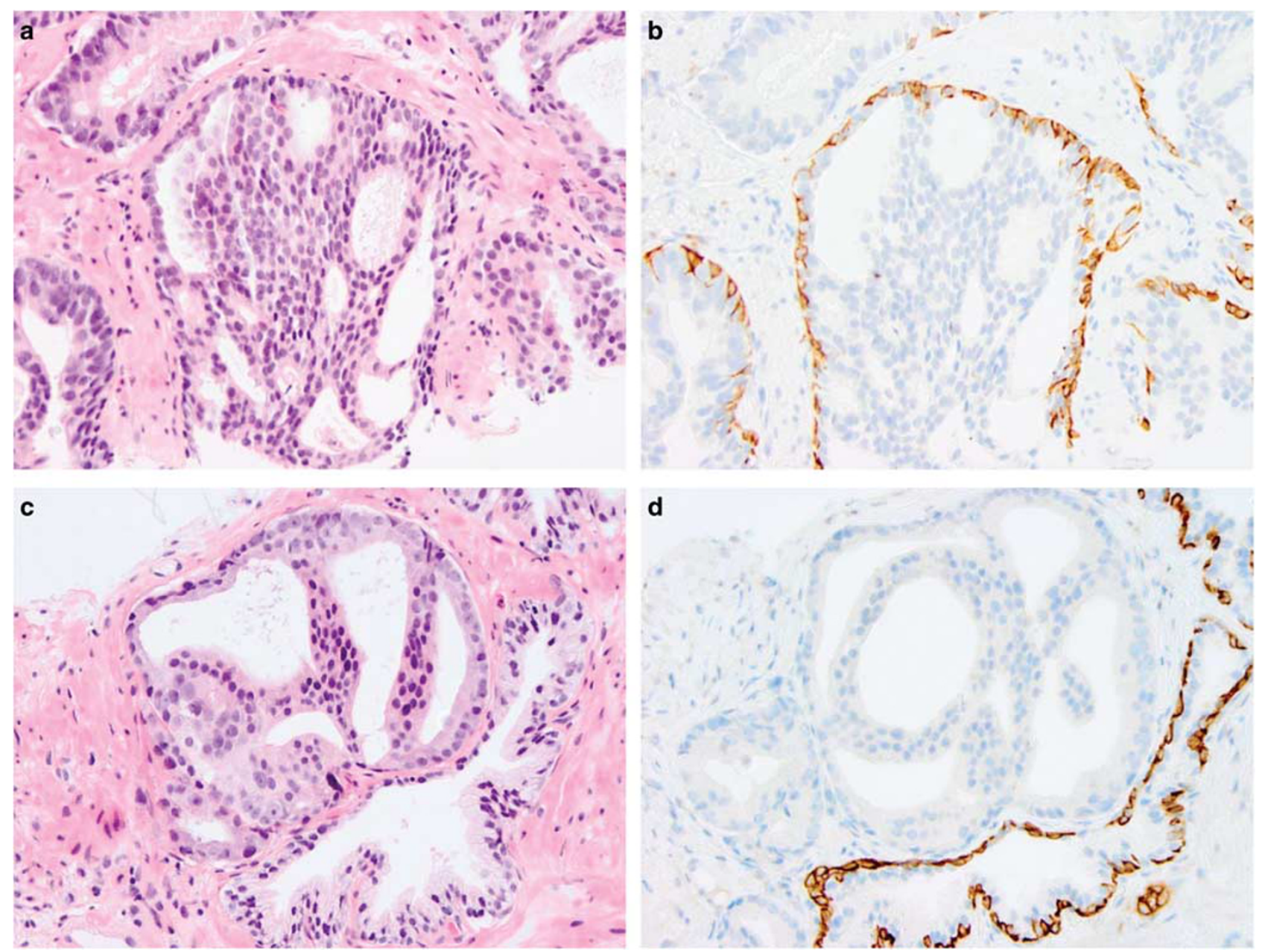

Figure 1 Intraductal carcinoma of the prostate consisting of a dilated gland filled with a malignant cribriform epithelial proliferation with a continuous pre-existent basal cell layer (a and b). Invasive cribriform Gleason grade 4 prostate cancer consists of a malignant cribriform proliferation without basal cell layer (c and d). Hematoxylin and eosin (HE; a and c), high-molecular-weight keratin (34BE12; b and d). Original magnifications, x200. 
time of diagnosis $(n=23)$, we selected patients with overall biopsy GS $3+4=7$ for the current study $(n=370)$.

\section{Pathological Evaluation}

Three investigators (CK, IK, GvL), who were blinded to patient information and outcome, revised all pathological slides in common sessions. For each biopsy core we recorded GS according to the 2014 International Society of Urological Pathology (ISUP) recommendations, total biopsy length, total tumor length, estimated Gleason grade 4 tumor percentage, presence of invasive cribriform pattern, and intraductal carcinoma. ${ }^{2}$ Since distinction of invasive cribriform and intraductal carcinoma lacks clinical relevance in the majority of cases and might be difficult, if not impossible without the use of immunohistochemistry, we labeled both patterns as one group (CR/IDC) as was also suggested by the ISUP 2014 consensus conference ${ }^{2,7}$ (Figure 1). Mean tumor percentage per patient was defined as the sum of total tumor length $(\mathrm{mm})$ divided by the sum of total biopsy length (mm). \%GG4 was determined by dividing the total length of invasive Gleason grade 4 and intraductal carcinoma by the total tumor length in all biopsies.

\section{Clinical Follow-up}

After diagnosis and initial treatment, patients were semiannually monitored by chart review to assess potential progression and secondary treatments. BCR was defined as prostate-specific antigen (PSA) levels of $\geq 0.2 \mathrm{ng} / \mathrm{ml}$ assessed at two consecutive time points $>3$ months apart after radical prostatectomy, or any PSA increase $>2 \mathrm{ng} / \mathrm{ml}$ higher than the PSA nadir value after radiation therapy. ${ }^{14}$

\section{Statistical Analysis}

For comparison of \%GG4 with clinical and pathologic parameters, we grouped the cases as follows: $>0 \%$ and $<10 \%, \geq 10 \%$, and $<25 \%$, and $\geq 25 \%$ and $<50 \%$ Gleason grade 4 pattern. Continuous parameters were analyzed by the Kruskal-Wallis test, categorical parameters by the Pearson's $\chi^{2}$ test. We estimated survival probabilities using the Kaplan-Meier method. Unadjusted comparisons for survival time were made using log-rank tests with censoring of men lost to follow-up. Crude and adjusted hazard ratios (HRs) for survival time were calculated using Cox proportional hazards regression. All statistical analyses were performed in SPSS version 21 (IBM, Chicago, IL, USA). Two-sided $P$-values of $<0.05$ were considered statistically significant.

\section{Results}

\section{Patient Characteristics}

A total of 370 patients with overall biopsy GS $3+4=7$ prostate cancer were identified (Table 1). One hundred and twenty-one patients (33\%) had $<10 \%$ GG4 component, 131 men (35\%) had $10-25 \%$ GG4 and 118 (32\%) had 25-50\% GG4. Age $(P=0.001)$, PSA $(P<0.001)$ and biopsy tumor volume $(P=0.001)$ were all significantly higher in tumors with a greater \%GG4. The mean percentage of positive biopsy cores was higher in men with $<10 \%$ GG4, in whom only four patients had one positive biopsy core $(P=0.01)$.

The primary therapeutic interventions for the entire cohort were radical prostatectomy $(n=146$; $39 \%)$, radiation therapy $(n=195 ; 53 \%)$, watchful waiting $(n=25 ; 7 \%)$ and endocrine therapy $(n=3$; $1 \%)$. Radiation therapy was performed more often

Table 1 Clinical and pathologic characteristics of Gleason score $3+4=7$ biopsies

\begin{tabular}{|c|c|c|c|c|}
\hline \multirow{2}{*}{ Parameter } & \multicolumn{3}{|c|}{ Percentage Gleason grade 4} & \multirow{2}{*}{$\mathrm{P}$-value } \\
\hline & $0-10 \%$ & $10-25 \%$ & $25-50 \%$ & \\
\hline Number & 121 & 131 & 118 & \\
\hline Age (years) & $65(66 ; 61-70)$ & $67(68 ; 63-72)$ & $68(69 ; 65-72)$ & 0.001 \\
\hline PSA (ng/ml) & $7.8(5.2 ; 3.7-7.1)$ & $9.2(5.9 ; 4.2-9.0)$ & $11.7(8.5 ; 5.4-13.4)$ & $<0.001$ \\
\hline$\%$ Positive biopsies & $51(50 ; 33-67)$ & $44(43 ; 29-57)$ & $48(43 ; 29-67)$ & 0.01 \\
\hline$\%$ Tumor volume & $39(37 ; 25-52)$ & $44(45 ; 27-59)$ & $50(51 ; 34-65)$ & 0.001 \\
\hline CR/IDC & $7(6 \%)$ & $29(22 \%)$ & $52(44 \%)$ & $<0.001$ \\
\hline \multicolumn{5}{|l|}{ Therapy } \\
\hline Radical prostatectomy & $58(48 \%)$ & $50(38 \%)$ & $38(32 \%)$ & 0.04 \\
\hline Radiation therapy & $52(43 \%)$ & $71(54 \%)$ & $72(61 \%)$ & 0.02 \\
\hline Endocrine therapy & $1(1 \%)$ & $2(2 \%)$ & 0 & 0.41 \\
\hline Watchful waiting & $9(7 \%)$ & $8(6 \%)$ & $8(7 \%)$ & 0.92 \\
\hline Unknown & $1(1 \%)$ & 0 & 0 & \\
\hline Disease-specific death & $4(3 \%)$ & $6(5 \%)$ & $13(11 \%)$ & 0.02 \\
\hline
\end{tabular}

Abbreviations: CR/IDC, invasive cribriform and/or intraductal carcinoma; PSA, prostate-specific antigen.

Mean (median; IQR) or absolute number (\%) are given. 
Table 2 Crude and adjusted Cox regression analysis for time to biochemical recurrence after radical prostatectomy

\begin{tabular}{|c|c|c|c|c|}
\hline & Univariate HR (95\% CI) & P-value & Multivariate HR (95\% CI) & P-value \\
\hline Age (years) & $1.03(0.95-1.11)$ & 0.49 & $1.03(0.95-1.11)$ & 0.54 \\
\hline PSA & $1.03(0.98-1.09)$ & 0.31 & $1.01(0.95-1.08)$ & 0.69 \\
\hline Percentage positive biopsies & $3.68(0.85-15.95)$ & 0.08 & $2.27(0.51-10.09)$ & 0.28 \\
\hline Tumor volume & $2.20(0.50-9.76)$ & 0.30 & $1.71(0.34-8.50)$ & 0.51 \\
\hline Percentage GG4 & $1.01(0.99-1.04)$ & 0.29 & $1.00(0.97-1.03)$ & 0.80 \\
\hline CR/IDC & $2.72(1.33-5.95)$ & 0.006 & $2.40(1.03-5.60)$ & 0.04 \\
\hline
\end{tabular}

Abbreviations: CI, confidence interval; CR/IDC, invasive cribriform and/or intraductal carcinoma; HR, hazard ratio; PSA, prostate-specific antigen.

Table 3 Crude and adjusted Cox regression analysis for time to biochemical recurrence after radiation therapy

\begin{tabular}{|c|c|c|c|c|}
\hline & Univariate HR $(95 \%$ CI) & $\mathrm{P}$-value & Multivariate HR (95\% CI) & $\mathrm{P}$-value \\
\hline Age (years) & $1.00(0.95-1.04)$ & 0.90 & $1.00(0.95-1.06)$ & 0.89 \\
\hline PSA & $1.05(1.04-1.07)$ & $<0.001$ & $1.03(1.01-1.04)$ & 0.001 \\
\hline Number positive biopsies & $20.10(7.70-52.51)$ & $<0.001$ & $8.55(2.78-26.32)$ & $<0.001$ \\
\hline Tumor volume & $7.55(2.69-21.23)$ & $<0.001$ & $3.24(1.00-10.53)$ & 0.05 \\
\hline Percentage GG4 & $1.02(1.01-1.04)$ & 0.009 & $1.01(0.99-1.04)$ & 0.19 \\
\hline CR/IDC & $2.73(1.72-4.35)$ & $<0.001$ & $1.20(0.68-2.13)$ & 0.53 \\
\hline
\end{tabular}

Abbreviations: CI, confidence interval; CR/IDC, invasive cribriform and/or intraductal carcinoma; HR, hazard ratio; PSA, prostate-specific antigen.

$(P=0.02)$ and radical prostatectomy less frequently $(P=0.04)$ in patients with higher \%GG4. Prostate cancer-specific death occurred in $4(3 \%), 6(5 \%)$ and $13(11 \%)$ men with $<10 \%, 10-25 \%$ and $25-50 \%$ GG4 component, respectively (log rank, $P=0.02$ ).

Invasive cribriform and/or intraductal carcinoma was observed in 88 GS $3+4=7$ patients $(24 \%)$. Invasive cribriform and/or intraductal carcinoma was present in $7 / 121(6 \%)$ men with $<10 \%$ GG4, in $29 / 131(22 \%)$ men with $10-25 \%$ GG4 and $52 / 118$ $(44 \%)$ men with $25-50 \%$ GG4 pattern $(P<0.001)$. Mean PSA level in men with invasive cribriform and/or intraductal carcinoma was $12.9 \mathrm{ng} / \mathrm{ml}$ (median 5.2; IQR $8.7-13.7 \mathrm{ng} / \mathrm{ml}$ ) and $8.5 \mathrm{ng} / \mathrm{ml}$ (median 4.0; IQR $5.8-8.7 \mathrm{ng} / \mathrm{ml})$ in men without $(P=0.001)$. Biopsy tumor volume was $56 \%$ (median $40 \%$; IQR $55-70 \%$ ) and $41 \%$ (median $23 \%$; IQR $39-56 \%$ ) in men with and without invasive cribriform and/or intraductal carcinoma $(P<0.001)$, respectively. Mean \%GG4 was 28\% (median 14\%; IQR 24-33\%) in men with invasive cribriform and/or intraductal carcinoma and $16 \%$ (median $6 \%$; IQR $12-24 \%$ ) in those without $(P<0.001)$.

\section{Radical Prostatectomy}

The mean follow-up after radical prostatectomy was 14.6 years (median 15.5; IQR 14.0-17.2 years), with 35/146 (24\%) men experiencing BCR after 5.8 years (median 4.4; IQR 2.0-9.2 years). BCR occurred more often in men with $10-25 \%$ (log rank, $P=0.04$ ) and $25-50 \%$ GG4 (log rank, $P=0.03$ ) than in those with $<10 \%$ GG4, but was not statistically different between the three groups (log rank for trend, $P=0.08$ ). The presence of invasive cribriform and/ or intraductal carcinoma was associated with postoperative BCR (log rank, $P=0.004$ ). In bivariate Cox regression analysis, invasive cribriform and/or intraductal carcinoma (HR 2.73; 95\% CI: 1.22-6.10; $P=0.04)$ was associated with BCR-free survival (BCRFS), whereas \%GG4 as continuous parameter (HR 1.00; 95\% CI: 0.98-1.03; $P=0.99$ ) was not. Adjusted analysis for age, PSA, percentage positive biopsies, tumor volume revealed invasive cribriform and/or intraductal carcinoma $(P=0.04)$ as the only independent parameter for BCRFS after radical prostatectomy (Table 2).

Since recent surveillance protocols include GS 3 $+4=7$ patients with low amounts of GG4 pattern, we also analyzed the predictive value of dichotomized \%GG4. ${ }^{15,16}$ In crude regression analysis, men with $10-50 \%$ GG4 were at elevated risk for BCRFS as compared with those < $10 \%$ GG4 (HR 2.43; 95\% CI: $1.10-5.37 ; P=0.03)$. In bivariate analysis, the presence of invasive cribriform and/or intraductal carcinoma (HR 2.33; 95\% CI: 1.12-4.84; $P=0.02$ ) was predictive for BCRFS, while 10-50\% GG4 did not meet conventional measures of statistical significance (HR 2.12; 95\% CI: 0.95-4.74; $P=0.07$ ).

\section{Radiation Therapy}

The mean follow-up after radiation therapy was 11.9 years (median 13.1; IQR 8.4-15.9 years). In total, 72 out of $195(37 \%)$ men experienced BCR after 5.7 years (median 4.9; IQR 3.4-7.6 years). BCR occurred more frequently in patients with higher \%GG4 (logrank test for trend, $P=0.02$ ) and in men with invasive cribriform and/or intraductal carcinoma (log rank, $P<0.001$ ). In bivariate Cox regression analysis, 
invasive cribriform and/or intraductal carcinoma (HR 2.43; 95\% CI: $1.49-4.00 ; P<0.001$ ), but not \% GG4 (HR 1.01; 95\% CI: $1.00-1.03 ; P=0.14$ ) was associated with BCRFS. Adjusted analysis showed that PSA $(P=0.001)$, number of positive biopsies $(P<0.001)$ and tumor volume $(P=0.05)$ were independently predictive BCRFS after radiation therapy, while \%GG4 $(P=0.19)$ and invasive cribriform and/or intraductal carcinoma $(P=0.53)$ were not (Table 3). Dichotomization of \%GG4 revealed no statistically significant difference in BCRFS between men with $<10 \%$ and 10-50\% GG4 (HR 1.67; 95\% CI: $0.93-3.00 ; P=0.09$ ). Bivariate analysis showed that invasive cribriform and/or intraductal carcinoma (HR 2.58; 95\% CI: 1.59-4.21; $P<0.001$ ) was predictive for BCRFS, while $10-50 \%$ GG4 was not (HR 1.24; 95\% CI: 0.67-2.29; $P=0.49$ ).

\section{Discussion}

Recent studies have indicated that relative quantity of GG4 pattern and presence of invasive cribriform and/or intraductal carcinoma are promising parameters for risk stratification of GS $3+4=7$ prostate cancer patients. In this study, we demonstrated that increased GG4 pattern was strongly associated with the presence of invasive cribriform and/or intraductal carcinoma. Patients with $<10 \%$ GG4 had invasive cribriform and/or intraductal carcinoma in $6 \%$, while it was present in $44 \%$ of men with 25-50\% GG4. In bivariate analysis, invasive cribriform and/or intraductal carcinoma was an independent parameter for BCR after radical prostatectomy and radiation therapy, while \%GG4 as nominal or dichotomized parameter was not. In adjusted analysis, invasive cribriform and/or intraductal carcinoma was an independent parameter for BCR after radical prostatectomy, but not after radiation therapy. These results indicate that the worse outcome of men with high \%GG4 might be explained by more frequent presence of invasive cribriform and/or intraductal carcinoma in this group.

Our results on the clinical relevance of \%GG4 and invasive cribriform and/or intraductal carcinoma are in line with previous studies. In a large number of radical prostatectomies, Sauter et al. ${ }^{4}$ found that increased \%GG4 went together with BCR. While \% GG4 pattern in GS $3+4=7$ biopsies has been associated with adverse features at radical prostatectomy, it was not an independent predictive factor for postoperative BCR. ${ }^{17,18}$ Biopsy invasive cribriform growth and/or intraductal carcinoma are related to non-organ-confined disease as well as BCR after radical prostatectomy and radiation therapy. ${ }^{11,19-21}$ Choy et $a .^{5}$ found independent prognostic value of both \%GG4 and invasive cribriform architecture on radical prostatectomy for BCR. The discordance with the current study might be explained by the fact that we determined \%GG4 at diagnostic biopsies instead of radical prostatectomy specimens, and that Choy et al. ${ }^{5}$ did not include intraductal carcinoma in their analysis.

The clinical relevance of intraductal carcinoma, invasive cribriform carcinoma and percentage Gleason grade 4 is increasingly being acknowledged. The World Health Organization and ISUP recommend that presence of intraductal carcinoma is routinely mentioned in pathology reports and that \%GG4 is reported in GS 7 prostate cancer patients. ${ }^{2,22}$ Percentage GG4 is currently applied as a novel parameter for inclusion of GS $3+4=7$ prostate cancer patients in some active surveillance protocols. ${ }^{15,16}$ Yamamoto et $a .^{23}$ for instance found that GS $3+4=7$ patients with $<5 \%$ GG4 on surveillance did not experience metastasis. Although the presence of invasive cribriform growth and/or intraductal carcinoma has not been formally acknowledged yet as an exclusion criterion for surveillance protocols, it has been suggested to exclude patients with intraductal carcinoma from surveillance. ${ }^{24}$ The results of our study implicate that invasive cribriform growth and/ or intraductal carcinoma might be a more reliable factor for therapeutic stratification than \%GG4 only, and should be included in pathology reports.

Considerable interobserver variability exists in differentiating GS $3+4=7$ and GS 6 on biopsies. ${ }^{25-28}$ We expect that this variability is mainly present in cases with low \%GG4, which has been one of the rationales for including GS $3+4=7$ patients with low \%GG4 in surveillance protocols. ${ }^{15,16}$ Grading variability is most prominent in distinguishing ill-formed and fused GS 7 glands from tangentially sectioned GS 6 glands, while concordance on cribriform growth is generally much better. ${ }^{25,26}$ The reproducibility of cribriform growth further supports the potential relevance of invasive cribriform and/or intraductal carcinoma for therapeutic decision making.

In this study, we applied overall instead of highest GS $3+4=7$ as inclusion criterion. ${ }^{7}$ Since GS might differ between separate cores of the same biopsy session, the overall GS presumes that all biopsies are part of the same tumor. This means that a patient could have positive biopsies with GS $6,3+4=7,4$ $+3=7$ or $4+4=8$ as long as the total \%GG4 is $<50 \%$. In contrast, highest GS $3+4=7$ excludes patients with GS $4+3=7$ and GS $4+4=8$ in single biopsy cores. We have not selected for highest GS $3+4=7$ in this study since this would have excluded patients with overall $<50 \%$ GG4. Other groups have shown improved performance of overall versus highest GS in biopsies, challenging clinical practice to classify patients according to the highest GS. ${ }^{4,17}$

In this study, regression analysis of \%GG4 dichotomized at a cutoff 10\% performed better than \%GG4 as continuous parameter. Postoperative BCR between patients with $25-50 \%$ GG4 was not statistically significant from men 10-25\% GG4. Such a trend was also found by others, and is probably due to small sample size. ${ }^{18}$ Despite the lack of independent prognostic value of \%GG4, GG4 quantity might 
still be of interest for further studies. Since this study with long term follow-up was performed on sextant biopsies in 1990s, it is important to further elaborate on the predictive value of biopsy \%GG4 and invasive cribriform and/or intraductal carcinoma in the current era of multiple and MRI-targeted biopsies.

In conclusion, we demonstrate that increased \% GG4 is associated with a higher frequency of invasive cribriform and/or intraductal carcinoma in GS $3+4=7$ prostate cancer biopsies. Invasive cribriform and/or intraductal carcinoma is an independent parameter for BCR after radical prostatectomy, while \%GG4 is not. Therefore, stratification for invasive cribriform and/or intraductal carcinoma could be more reliable for inclusion of GS $3+4=7$ prostate cancer patients in active surveillance than \%GG4 alone. The presence of invasive cribriform and intraductal carcinoma should therefore routinely be included in pathology reports.

\section{Disclosure/conflict of interest}

The authors declare no conflict of interest.

\section{References}

1 Epstein JI, Allsbrook WC Jr, Amin MB et al. The 2005 International Society of Urological Pathology (ISUP) Consensus Conference on Gleason Grading of Prostatic Carcinoma. Am J Surg Pathol 2005;29:1228-1242.

2 Epstein JI, Egevad L, Amin MB, et al. The 2014 International Society of Urological Pathology (ISUP) Consensus Conference on Gleason Grading of Prostatic Carcinoma: definition of grading patterns and proposal for a new grading system. Am J Surg Pathol 2016;40: 244-252.

3 Vis AN, Roemeling S, Kranse R, et al. Should we replace the Gleason score with the amount of highgrade prostate cancer? Eur Urol 2007;51:931-939.

4 Sauter G, Steurer S, Clauditz TS, et al. Clinical utility of quantitative gleason grading in prostate biopsies and prostatectomy specimens. Eur Urol 2016;69:592-598.

5 Choy B, Pearce SM, Anderson BB, et al. Prognostic significance of percentage and architectural types of contemporary gleason pattern 4 prostate cancer in radical prostatectomy. Am J Surg Pathol 2016;40: 1400-1406.

6 Dong F, Yang P, Wang C, et al. Architectural heterogeneity and cribriform pattern predict adverse clinical outcome for Gleason grade 4 prostatic adenocarcinoma. Am J Surg Pathol 2013;37:1855-1861.

7 Kweldam CF, Kummerlin IP, Nieboer D, et al. Diseasespecific survival of patients with invasive cribriform and intraductal prostate cancer at diagnostic biopsy. Mod Pathol 2016;29:630-636.

8 Kweldam CF, Wildhagen MF, Steyerberg EW, et al. Cribriform growth is highly predictive for postoperative metastasis and disease-specific death in Gleason score 7 prostate cancer. Mod Pathol 2015;28:457-464.

9 Trudel D, Downes MR, Sykes J, et al. Prognostic impact of intraductal carcinoma and large cribriform carcinoma architecture after prostatectomy in a contemporary cohort. Eur J Cancer 2014;50: 1610-1616.

10 Kimura K, Tsuzuki T, Kato M, et al. Prognostic value of intraductal carcinoma of the prostate in radical prostatectomy specimens. Prostate 2014;74:680-687.

11 Van der Kwast T, Al Daoud N, Collette L, et al. Biopsy diagnosis of intraductal carcinoma is prognostic in intermediate and high risk prostate cancer patients treated by radiotherapy. Eur J Cancer 2012;48: 1318-1325.

12 Roobol MJ, Schroder FH. European Randomized Study of Screening for Prostate Cancer: achievements and presentation. BJU Int 2003;92(Suppl 2):117-122.

13 Schroder FH, Hugosson J, Roobol MJ, et al. Screening and prostate-cancer mortality in a randomized European study. N Engl J Med 2009;360:1320-1328.

14 Roach M 3rd, Hanks G, Thames H Jr., et al. Defining biochemical failure following radiotherapy with or without hormonal therapy in men with clinically localized prostate cancer: recommendations of the RTOG-ASTRO Phoenix Consensus Conference. Int J Radiat Oncol Biol Phys 2006;65:965-974.

15 Chen RC, Rumble RB, Loblaw DA, et al. Active Surveillance for the Management of Localized Prostate Cancer (Cancer Care Ontario Guideline): American Society of Clinical Oncology Clinical Practice Guideline Endorsement. J Clin Oncol 2016;34:2182-2190.

16 Morash C, Tey R, Agbassi C, et al. Active surveillance for the management of localized prostate cancer: Guideline recommendations. Can Urol Assoc J 2015;9: 171-178.

17 Cole AI, Morgan TM, Spratt DE, et al. Prognostic value of percent Gleason grade 4 at prostate biopsy in predicting prostatectomy pathology and recurrence. J Urol 2016;196:405-411.

18 Perlis N, Sayyid R, Evans A, et al. Limitations in predicting organ-confined prostate cancer in patients with Gleason pattern 4 on biopsy: Implications for active surveillance. J Urol 2017;197:75-83.

19 Guo CC, Epstein JI. Intraductal carcinoma of the prostate on needle biopsy: Histologic features and clinical significance. Mod Pathol 2006;19:1528-1535.

20 Flood TA, Schieda N, Keefe DT, et al. Utility of Gleason pattern 4 morphologies detected on transrectal ultrasound (TRUS)-guided biopsies for prediction of upgrading or upstaging in Gleason score $3+4=7$ prostate cancer. Virchows Arch 2016;469:313-319.

21 Keefe DT, Schieda N, El Hallani S, et al. Cribriform morphology predicts upstaging after radical prostatectomy in patients with Gleason score $3+4=7$ prostate cancer at transrectal ultrasound (TRUS)-guided needle biopsy. Virchows Arch 2015;467:437-442.

22 Humphrey PA, Moch H, Cubilla AL, et al. The 2016 WHO Classification of Tumours of the Urinary System and Male Genital Organs-Part B: prostate and bladder tumours. Eur Urol 2016;70:106-119.

23 Yamamoto T, Musunuru B, Vesprini D, et al. Metastatic prostate cancer in men initially treated with active surveillance. J Urol 2016;195:1409-1414.

24 Amin MB, Lin DW, Gore JL, et al. The critical role of the pathologist in determining eligibility for active surveillance as a management option in patients with prostate cancer: consensus statement with recommendations supported by the College of American Pathologists, International Society of Urological Pathology, Association of Directors of Anatomic and Surgical Pathology, the New Zealand Society of Pathologists, 
and the Prostate Cancer Foundation. Arch Pathol Lab Med 2014;138:1387-1405.

25 Egevad L, Ahmad AS, Algaba F, et al. Standardization of Gleason grading among 337 European pathologists. Histopathology 2013;62:247-256.

26 Kweldam CF, Nieboer D, Algaba F, et al. Gleason Grade 4 prostate adenocarcinoma patterns: an inter-observer agreement study among genitourinary pathologists. Histopathology 2016;69:441-449.
27 McKenney JK, Simko J, Bonham M, et al. The potential impact of reproducibility of Gleason grading in men with early stage prostate cancer managed by active surveillance: a multi-institutional study. J Urol 2011;186:465-469.

28 Rodriguez-Urrego PA, Cronin AM, Al-Ahmadie HA, et al. Interobserver and intraobserver reproducibility in digital and routine microscopic assessment of prostate needle biopsies. Hum Pathol 2011;42:68-74. 